\title{
Momentum space metric, non-local operator, and topological insulators
}

\author{
Shunji Matsuura ${ }^{1}$ and Shinsei Ryu ${ }^{2}$ \\ ${ }^{1}$ Kavli Institute for Theoretical Physics, University of California, Santa Barbara, CA 93106, USA \\ ${ }^{2}$ Department of Physics, University of California, Berkeley, CA 94720, USA
}

(Dated: October 29, 2018)

\begin{abstract}
Momentum space of a gapped quantum system is a metric space: it admits a notion of distance reflecting properties of its quantum ground state. By using this quantum metric, we investigate geometric properties of momentum space. In particular, we introduce a non-local operator which represents distance square in real space and show that this corresponds to the Laplacian in curved momentum space, and also derive its path integral representation in momentum space. The quantum metric itself measures the second cumulant of the position operator in real space, much like the Berry gauge potential measures the first cumulant or the electric polarization in real space. By using the non-local operator and the metric, we study some aspects of topological phases such as topological invariants, the cumulants and topological phase transitions. The effect of interactions to the momentum space geometry is also discussed.
\end{abstract}

PACS numbers: 72.10.-d,73.21.-b,73.50.Fq

\section{INTRODUCTION}

Topological insulators (superconductors) have been attracting a lot of interest both theoretically and experimentally. Similar to trivial insulators, they have a band gap in the bulk. The striking feature that distinguishes topological insulators (superconductors) from trivial ones is that they have gapless modes on the boundaries. In other words, boundaries of topological insulators are totally metallic. These gapless modes are robust under perturbations and cannot be gapped without going through a quantum phase transition, i.e., the gapless modes are topologically protected. One of the best-known examples of the topological phases is the integer quantum hall effect (IQHE) in 2+1 dimensions where the Hall conductance $\sigma_{x y}$ is quantized.

Recent excitement on topological phases comes from the discoveries of new topological phases; the quantum spin Hall effect (QSHE) in two space dimensions and the $\mathbb{Z}_{2}$ topological insulator in three space dimensions [1] 8. The existence of these new phases suggests that the topological phases are relatively generic for electron systems: they are not restricted to two space dimensions nor they do not necessarily require time reversal symmetry breaking.

Following these discoveries, the exhaustive classification of topological insulators and superconductors of noninteracting fermionic systems was proposed in Refs. 9 11. The classification is based on discrete symmetries that are generic in quantum systems; time reversal symmetry, charge conjugation and chiral (sublattice) symmetry. All the topological phases are characterized by certain types of K-theory charges; $\mathbb{Z}$ Chern/winding numbers and $\mathbb{Z}_{2}$ numbers. (For an introduction of K-theory charge for fermi surfaces, see 12].) These quantities are defined in momentum space: the Chern number is associated with the Berry curvature (gauge potential), the winding number is given by a Wess-Zumino-Witten type term constructed from a projection operator, and $\mathbb{Z}_{2}$ charge is given by some gauge invariant product of time reversal polarization at time reversal invariant points. The well-known example is again the IQHE: the Hall conductance $\sigma_{x y}$ is given by the first Chern number of the momentum space Berry curvature via the TKNN formula 13, 14. For time reversal symmetric systems, Berry curvature does not give a non-trivial Chern number. Instead, the $\mathbb{Z}_{2}$ number, which basically counts the number of Kramers pairs or Dirac points, gives the topological charge [1 $13,6,15]$.

It is natural to ask if there are other functions or quantities in momentum space that may also play a role. It is known that the Berry gauge potential is not the only quantity that we can define in momentum space. For instance, we can generalize the idea of the Berry gauge potential to higher order tensors such as a metric. Given the fact that topological invariants constructed out of metric play a role in general relativity, it would be interesting to investigate physical properties of the momentum space metric to see if it captures new aspects of topological phases. The momentum space metric (the quantum metric, or the Bures metric) has considered in Refs. 16, 17] in the context of localization and polarization of Wannier functions and a relation to the cumulants. In this paper, we explore a connection between the momentum space metric and physically observable quantities. Especially we propose a non-local operator that corresponds to the Laplacian in curved momentum space. We will also study some representatives of topological insulators from the point of view of the momentum space metric.

The organization of this paper is as follows. In section [1, we review the construction of the Berry gauge potential and the Bures metric (the quantum metric). In section III, we first review the known properties of the Bures metric and then define and investigate a nonlocal operator corresponding to the Laplacian in curved momentum space. In section [V] we study several topological phases by using the Bures metric, especially for symmetry classes A (unitary) and AII (symplectic, spin- 
orbit).

\section{MOMENTUM SPACE GAUGE FIELD AND GRAVITY}

\section{A. setup}

In this section, we will explore momentum space geometry and topology by studying gauge field (the Berry gauge field) and metric (the Bures metric). Consider a tight-binding Hamiltonian,

$$
H=\sum_{\mathbf{r}, \mathbf{r}^{\prime}} \psi^{\dagger}(\mathbf{r}) \mathcal{H}\left(\mathbf{r}, \mathbf{r}^{\prime}\right) \psi\left(\mathbf{r}^{\prime}\right)
$$

where $\psi(\mathbf{r})$ is an $N_{f}$-component fermion annihilation operator, and index $\mathbf{r}$ labels a site on a $d$-dimensional lattice (the internal indices are suppressed). Each block in the single particle Hamiltonian $\mathcal{H}\left(\mathbf{r}, \mathbf{r}^{\prime}\right)$ is an $N_{f} \times N_{f}$ matrix, satisfying the hermiticity condition $\mathcal{H}^{\dagger}\left(\mathbf{r}^{\prime}, \mathbf{r}\right)=\mathcal{H}\left(\mathbf{r}, \mathbf{r}^{\prime}\right)$. and we assume the total size of the single particle Hamiltonian is $N_{f} V \times N_{f} V$, where $V$ is the total number of lattice sites. The components in $\psi(\mathbf{r})$ can describe, e.g., orbitals or spin degrees of freedom, as well as different sites within a crystal unit cell centered at $\mathbf{r}$.

Provided the system has translational symmetry,

$$
\mathcal{H}\left(\mathbf{r}, \mathbf{r}^{\prime}\right)=\mathcal{H}\left(\mathbf{r}-\mathbf{r}^{\prime}\right)
$$

with periodic boundary conditions in each spatial direction (i.e., the system is defined on a torus $T^{d}$ ), we can perform the Fourier transformation and obtain in momentum space

$$
H=\sum_{\mathbf{k} \in \mathrm{BZ}} \psi^{\dagger}(\mathbf{k}) \mathcal{H}(\mathbf{k}) \psi(\mathbf{k})
$$

where the crystal momentum $\mathbf{k}$ runs over the first Brillouin zone (BZ), and the Fourier component of the fermion operator and the Hamiltonian are given by

$$
\psi(\mathbf{r})=V^{\frac{-1}{2}} \sum_{\mathbf{k} \in \mathrm{BZ}} e^{i \mathbf{k} \cdot \mathbf{r}} \psi(\mathbf{k}), \mathcal{H}(\mathbf{k})=\sum_{\mathbf{r}} e^{-i \mathbf{k} \cdot \mathbf{r}} \mathcal{H}(\mathbf{r})
$$

where $V$ is the total number of sites, and $\mathcal{H}(\mathbf{k})$ is an $N_{f} \times N_{f}$ matrix.

The Bloch Hamiltonian $\mathcal{H}(\mathbf{k})$ is diagonalized by

$$
\mathcal{H}(\mathbf{k})\left|u^{a}(\mathbf{k})\right\rangle=E^{a}(\mathbf{k})\left|u^{a}(\mathbf{k})\right\rangle, \quad a=1, \ldots, N_{f},
$$

where and $\left|u^{a}(\mathbf{k})\right\rangle$ is the $a$-th Bloch wavefunction with energy $E^{a}(\mathbf{k})$. The fermion operator can be expanded in terms of the eigen functions as

$$
\psi_{i}(\mathbf{k})=u_{i}{ }^{a}(\mathbf{k}) \chi_{a}(\mathbf{k}), \quad i, a=1, \ldots, N_{f},
$$

where $\chi_{a}(\mathbf{k})$ represents a fermionic operator in the eigen basis and given by

$$
\chi_{b}(\mathbf{k})=\left[u_{i}^{b}(\mathbf{k})\right]^{*} \psi_{i}(\mathbf{k}) .
$$

We assume that there is a finite gap at the Fermi level, and therefore we obtain a unique ground state $|\Phi(\mathbf{k})\rangle$ for each $\mathbf{k}$ by filling all states below the Fermi level. (In this paper, we always adjust $E^{\hat{a}}(\mathbf{k})$ in such a way that the Fermi level is at zero energy.) We assume there are $N_{-}\left(N_{+}\right)$occupied (unoccupied) Bloch wavefunctions for each $\mathbf{k}$ with $N_{+}+N_{-}=N_{f}$. We call the set of filled Bloch wavefunctions $\left\{\left|u_{\hat{a}}^{-}(\mathbf{k})\right\rangle\right\}$, where hatted indices $\hat{a}=$ $1, \ldots, N_{-}$labels the occupied bands only. The manybody ground state $|\Phi\rangle$ is the filled Fermi sea

$$
|\Phi\rangle=\bigotimes_{\mathbf{k} \in \mathrm{BZ}}|\Phi(\mathbf{k})\rangle, \quad|\Phi(\mathbf{k})\rangle=\prod_{\hat{a}=1}^{N_{-}} \chi_{\hat{a}}^{\dagger}(\mathbf{k})|0\rangle .
$$

\section{B. Berry connection and Bures metric (quantum metric)}

The momentum space gauge field and metric can be introduced by comparing two states $\left|\Phi\left(\mathbf{k}_{1}\right)\right\rangle$ and $\left|\Phi\left(\mathbf{k}_{2}\right)\right\rangle$ at different momenta $\mathbf{k}_{1,2}$. From the overlap $\langle\Phi(\mathbf{k})| \Phi(\mathbf{k}+$ $d \mathbf{k})\rangle$, we define the Berry connection 18, 19 $\mathcal{A}(\mathbf{k})=$ $A_{\mu}(\mathbf{k}) d k_{\mu}$ by the expansion

$$
\langle\Phi(\mathbf{k}) \mid \Phi(\mathbf{k}+d \mathbf{k})\rangle=1+A_{\mu}(\mathbf{k}) d k_{\mu}+\cdots,
$$

where $\mu=1, \cdots, d$. Similarly, to define the momentum space metric, we consider the quantum distance $D_{\mathbf{k}_{1}, \mathbf{k}_{2}}$ between two states $\left|\Phi\left(\mathbf{k}_{1}\right)\right\rangle$ and $\left|\Phi\left(\mathbf{k}_{2}\right)\right\rangle$ by $D_{\mathbf{k}_{1}, \mathbf{k}_{2}}^{2}=$ $1-\left|\left\langle\Phi\left(\mathbf{k}_{1}\right) \mid \Phi\left(\mathbf{k}_{2}\right)\right\rangle\right|^{2} \quad 20$. When these two states are infinitesimally close to each other, $\mathbf{k}_{1}=\mathbf{k}, \mathbf{k}_{2}=\mathbf{k}+d \mathbf{k}$, the quantum distance can be expanded as

$$
|\langle\Phi(\mathbf{k}) \mid \Phi(\mathbf{k}+d \mathbf{k})\rangle|^{2}=1-g_{\mu \nu}(\mathbf{k}) d k_{\mu} d k_{\nu}+\cdots .
$$

This defines the quantum metric $g_{\mu \nu}(\mathbf{k})$.

For the case when $|\Phi(\mathbf{k})\rangle$ is given by a Slater determinant, the overlap $\left\langle\Phi\left(\mathbf{k}_{1}\right) \mid \Phi\left(\mathbf{k}_{2}\right)\right\rangle$ is computed as a determinant of an $N_{-} \times N_{-}$matrix made of Bloch states,

$$
\begin{aligned}
\left\langle\Phi\left(\mathbf{k}_{1}\right) \mid \Phi\left(\mathbf{k}_{2}\right)\right\rangle & =\operatorname{det} S\left(\mathbf{k}_{1}, \mathbf{k}_{2}\right), \\
S_{\hat{a} \hat{b}}\left(\mathbf{k}_{1}, \mathbf{k}_{2}\right) & :=\left\langle u_{\hat{a}}^{-}\left(\mathbf{k}_{1}\right) \mid u_{\hat{b}}^{-}\left(\mathbf{k}_{2}\right)\right\rangle
\end{aligned}
$$

where $\left|u_{\hat{a}}^{-}(\mathbf{k})\right\rangle\left(\hat{a}=1, \cdots, N_{-}\right)$, are the Bloch wavefunction for filled bands. Hence the Berry connection and the Bures metric are given by

$$
\begin{aligned}
A_{\mu}(\mathbf{k})= & \left\langle u_{\hat{a}}^{-}(\mathbf{k}) \mid \partial_{\mu} u_{\hat{a}}^{-}(\mathbf{k})\right\rangle \\
g_{\mu \nu}(\mathbf{k})= & \frac{1}{2}\left[\left\langle\partial_{\mu} u_{\hat{a}}^{-} \mid \partial_{\nu} u_{\hat{a}}^{-}\right\rangle+\left\langle\partial_{\nu} u_{\hat{a}}^{-} \mid \partial_{\mu} u_{\hat{a}}^{-}\right\rangle\right. \\
& \left.\quad+2\left\langle u_{\hat{a}}^{-} \mid \partial_{\mu} u_{\hat{b}}^{-}\right\rangle\left\langle u_{\hat{b}}^{-} \mid \partial_{\nu} u_{\hat{a}}^{-}\right\rangle\right] \\
= & \operatorname{Re}\left\langle\partial_{\mu} u_{\hat{a}}^{-} \mid \partial_{\nu} u_{\hat{a}}^{-}\right\rangle-\left\langle\partial_{\mu} u_{\hat{a}}^{-} \mid u_{\hat{b}}^{-}\right\rangle\left\langle u_{\hat{b}}^{-} \mid \partial_{\nu} u_{\hat{a}}^{-}\right\rangle,
\end{aligned}
$$

where repeated indices are summed, and $\partial_{\mu}=\partial_{k_{\mu}}$, etc.

For the case with more than one occupied bands, one can also introduce the non-Abelian Berry connection by

$$
\mathcal{A}^{\hat{a} \hat{b}}(\mathbf{k})=A_{\mu}^{\hat{a} \hat{b}}(\mathbf{k}) d k_{\mu}=\left\langle u_{\hat{a}}^{-}(\mathbf{k}) \mid d u_{\hat{b}}^{-}(\mathbf{k})\right\rangle,
$$


where $A_{\mu}^{\hat{a} \hat{b}}=-\left(A_{\mu}^{\hat{b} \hat{a}}\right)^{*}$. A non-Abelian generalization of the metric is discussed in Refs. 21.

The above formula for the gauge field and metric can compactly summarized in terms the spectral projector onto the filled Bloch states and the "Q-matrix", which are defined by

$$
P(\mathbf{k}):=\sum_{\hat{a}}\left|u_{\hat{a}}^{-}(\mathbf{k})\right\rangle\left\langle u_{\hat{a}}^{-}(\mathbf{k})\right|, Q(\mathbf{k}):=1-2 P(\mathbf{k}) .
$$

Then, the U(1) part of the Berry curvature the metric are given by

$$
\begin{aligned}
F_{\mu \nu}(\mathbf{k}) & =F_{\mu \nu}^{\hat{a} \hat{a}}(\mathbf{k})=\frac{1}{4} \operatorname{tr}\left[Q \partial_{\nu} Q \partial_{\mu} Q\right], \\
g_{\mu \nu}(\mathbf{k}) & =\frac{1}{8} \operatorname{tr}\left[\partial_{\mu} Q \partial_{\nu} Q\right] .
\end{aligned}
$$

I.e., $g_{\mu \nu}$ and $F_{\mu \nu}$ are the symmetric and antisymmetric part of $\operatorname{tr}\left[P \partial_{\nu} P \partial_{\mu} P\right]$, respectively, $2 g_{\mu \nu}+F_{\mu \nu}=$ $-\operatorname{tr}\left[P \partial_{\nu} P \partial_{\mu} P\right]$.

\section{NON-LOCAL OPERATORS AND MOMENTS OF THE POSITION OPERATOR}

In this section, we discuss the expectation values of the position operator, and its second cumulant in a quantum ground state. For band insulators, the Berry connection and the Bures metric naturally enter in evaluating these expectation values. This is to some extent expected, since the definition of the Berry connection and the Bures metric involves a derivative with respect to momentum, this in turn suggests that it is related to the position operator in real space. We will first review these ideas developed in the theory of macroscopic polarization, and in the context of maximally localized Wannier functions in solids. We will then introduce yet another formalism for the second cumulant of the position operator, which is for the geometric, rather than arithmetic, mean of the second cumulant. We will show how it is related to the momentum space spectral geometry.

\section{A. Marzari-Vanderbilt theory}

Let us consider the following non-local operator:

$$
z_{\boldsymbol{\alpha}}=\exp \left[\sum_{\mathbf{r}} i \boldsymbol{\alpha} \cdot \mathbf{r} \rho(\mathbf{r})\right]
$$

where $\mathbf{r}=\left(x_{1}, x_{2}, \cdots, x_{d}\right)$ labels sites on the $d$ dimensional hyper cubic lattice with $N^{d}(=V)$ sites, and can be written, in terms of the lattice constant $\mathfrak{a}$ as

$$
x_{\mu}=n_{\mu} \times \mathfrak{a}, \quad n_{\mu}=1, \cdots, N, \quad L=\mathfrak{a} N .
$$

$\rho(\mathbf{r})$ is the density operator at $\mathbf{r}$,

$$
\rho(\mathbf{r})=\sum_{i=1}^{N_{f}} \psi_{i}^{\dagger}(\mathbf{r}) \psi_{i}(\mathbf{r})
$$

We now choose

$$
\boldsymbol{\alpha}=2 \pi \hat{\mathbf{n}}_{\mu} / L,
$$

where $\hat{\mathbf{n}}_{\mu}$ is the unit vector in the $\mu$-direction and consider the expectation value of the non-local operator,

$$
\mathfrak{z}_{\mu}:=\left\langle\Psi\left|z_{2 \pi \hat{\mathbf{n}}_{\mu} / L}\right| \Psi\right\rangle .
$$

The logarithm of $\mathfrak{z}$ defines the expectation of the position operator in periodic systems

$$
\left\langle x_{\mu}\right\rangle=\frac{L}{2 \pi} \operatorname{Im} \ln \mathfrak{z}_{\mu} .
$$

(The "single point formula" for the macroscopic polarization by Resta 22 24.)

For the case of Slater determinant, this can be evaluated as

$$
\left\langle x_{\mu}\right\rangle=\frac{L}{2 \pi} \sum_{\mathbf{k}} \operatorname{tr} \ln S\left(\mathbf{k}, \mathbf{k}+\hat{\mathbf{k}}_{\mu}\right)
$$

where $\hat{\mathbf{k}}_{\mu}=2 \pi \hat{\mathbf{n}}_{\mu} / L$ and the trace is taken over occupied states. In the continuum limit, this reduces to the Wilson loop of the $\mathrm{U}(1)$ part of the Berry connection, $\left\langle x_{\mu}\right\rangle \sim$ $\ln \exp \oint_{\mathrm{BZ}} \operatorname{tr} \mathcal{A}(\mathbf{k})$.

In general, we can consider the generating function

$$
C(\boldsymbol{\alpha})=\left\langle\Psi\left|z_{\boldsymbol{\alpha}}\right| \Psi\right\rangle \text {. }
$$

For slater determinants, this can be evaluated as

$$
\ln C(\boldsymbol{\alpha})=\sum_{\mathbf{k}} \operatorname{tr} \ln S(\mathbf{k}, \mathbf{k}+\boldsymbol{\alpha})
$$

Taking the derivative with respect to $\boldsymbol{\alpha}$, we generate all cumulants of the position operators,

$$
\begin{aligned}
& \left\langle x_{1}^{n_{1}} x_{2}^{n_{2}} \cdots x_{d}^{n_{d}}\right\rangle_{c} \\
& \left.\quad \simeq i^{n}\left(\frac{\partial^{n}}{\partial \alpha_{1}^{n_{1}} \partial \alpha_{2}^{n_{2}} \cdots \partial \alpha_{d}^{n_{d}}}\right) \ln C(\boldsymbol{\alpha})\right|_{\alpha=0} .
\end{aligned}
$$

where the derivative with respect to $\alpha_{\mu}$ should be properly discretized [24]. E.g.,

$$
\left\langle x_{\mu} x_{\mu}\right\rangle_{c}=\left(\frac{\mathfrak{a}}{2 \pi}\right)^{2} \frac{-2}{N_{f} L} \ln \prod_{\mathbf{k}} \operatorname{det}\left|S\left(\mathbf{k}, \mathbf{k}+\hat{\mathbf{k}}_{\mu}\right)\right|^{2},
$$

where $\mu$ is not summed. In the continuum, the second cumulant is given by

$$
\left\langle x_{\mu} x_{\nu}\right\rangle_{c}=\frac{v}{N_{f}(2 \pi)^{d}} \int_{\mathrm{BZ}} d \mathbf{k} g_{\mu \nu}(\mathbf{k})=: \frac{v}{N_{f}} \Omega_{I, \mu \nu} .
$$

with $v$ the cell volume.

This expression for the second cumulant should be compared with $\Omega=\sum_{\hat{a}}\left[\left\langle r^{2}\right\rangle_{\hat{a}}-\langle\mathbf{r}\rangle_{\hat{a}}^{2}\right]$, where $\left\langle r^{2}\right\rangle_{\hat{a}}=\left[v /(2 \pi)^{d}\right]\left\langle\partial_{\mu} u_{\hat{a}}(\mathbf{k}) \mid \partial_{\mu} u_{\hat{a}}(\mathbf{k})\right\rangle$ and $\langle\mathbf{r}\rangle_{\hat{a}}=$ $i\left[v /(2 \pi)^{d}\right] \int d \mathbf{k}\left\langle u_{\hat{a}}(\mathbf{k})\left|\nabla_{\mathbf{k}}\right| u_{\hat{a}}(\mathbf{k})\right\rangle$, which was also used in [16. In that paper, it was showed that in a band model, $\Omega$ is not gauge invariant and hence cannot directly be related to an observable, while $\Omega_{I}$ is gauge invariant. This gauge dependence argument would not be relevant in this paper. We mostly consider general continuum models. 


\section{B. non-local operator}

We introduce yet another non-local operator $\eta$ by

$$
\eta=\exp \left\{\sum_{\mathbf{r}} \Theta(\mathbf{r})[\rho(\mathbf{r})-\bar{\rho}]\right\},
$$

where $\bar{\rho}$ is the average density, and $\Theta(\mathbf{r})$ is assumed to be written in terms of a logarithm of some function,

$$
\Theta(\mathbf{r}):=\ln \theta(\mathbf{r}) \text {. }
$$

For our purpose, we will choose

$$
\begin{gathered}
\theta(\mathbf{r})=\left[-2 \sum_{\mu=1}^{d} \cos \frac{2 \pi n_{\mu}}{N}+2 d\right]^{\alpha} \\
\text { with } \quad \alpha=1 / N_{e}=1 /(f V)
\end{gathered}
$$

where $N_{e}$ is the total number of electrons, and $f=N_{e} / V$ is the filling fraction. By expanding the cosine in the decompactifying limit, $x_{\mu} / L \rightarrow 0$,

$$
\ln \theta(\mathbf{r}) \sim \ln \sum_{\mu=1}^{d}\left(\frac{2 \pi x_{\mu}}{\mathfrak{a} N}\right)^{2}=\ln \frac{r^{2}}{\mathfrak{a}^{2}}-\ln \left(\frac{2 \pi}{N}\right)^{2},
$$

and noting

$$
\begin{aligned}
& \int d^{d} r\left[\ln \frac{r^{2}}{\mathfrak{a}^{2}}-\ln \left(\frac{2 \pi}{N}\right)^{2}\right][\rho(\mathbf{r})-\bar{\rho}] \\
= & \int d^{d} r \ln \left(r^{2} / \mathfrak{a}^{2}\right)[\rho(\mathbf{r})-\bar{\rho}],
\end{aligned}
$$

where we have used the charge conservation $\int d^{d} r[\rho(\mathbf{r})-$ $\bar{\rho}]=0, \eta$ may be interpreted, in the continuum limit, as

$$
\eta=\exp \left\{\alpha \int \frac{d^{d} r}{\mathfrak{a}^{d}}\left(\ln r^{2} / \mathfrak{a}^{2}\right)[\rho(\mathbf{r})-\bar{\rho}]\right\} .
$$

In the first quantization, the density operator is given by

$$
\rho(\mathbf{r})=\sum_{i=1}^{N_{e}} \delta^{(d)}\left(\mathbf{r}-\mathbf{r}_{i}\right)
$$

where $\mathbf{r}_{i}$ is the position operator of the $i$-th particle. Then,

$$
\exp \alpha \int d^{d} r\left(\ln r^{2}\right) \rho(\mathbf{r})=e^{\alpha \sum_{i=1}^{N_{e}} \ln r_{i}^{2}}=\prod_{i=1}^{N_{e}}\left(r_{i}^{2}\right)^{\alpha}
$$

Thus, the expectation value of $\eta$ is nothing but the geometric mean of the position operator over $N_{e}$ fermions.

We note that by choosing $\Theta(\mathbf{r})$ in Eq. (29) properly, $\eta$ can represent several different non-local operators familiar in condensed matter context. When we choose

$$
\theta(\mathbf{r})=e^{i \frac{2 \pi n_{x}}{N}}, \quad \Theta(\mathbf{r})=i \frac{2 \pi n_{x}}{N}
$$

$\eta$ is the twist operator $\mathfrak{z}$ used in the theory of electric polarization. In two dimensions, and when we choose

$$
\theta(\mathbf{r})=\left(z-z_{0}\right)^{m}, \quad \Theta(\mathbf{r})=\ln \left(z-z_{0}\right)^{m},
$$

where $z=x+i y$, and $z_{0}$ is some reference point, then $\eta$ is the disorder operator studied by Shindou et al. 25 , 26. Yet another non-local operator in the form (29) is discussed in Ref. [27] in the context of the entanglement entropy.

\section{C. the non-local operator for band insulators}

We now specialize to band insulators and evaluate the expectation value of the non-local operator $\eta$. Below, we will evaluate the expectation value $\langle\Phi|\eta| \Phi\rangle$ for a band insulator. Since we can factorize $\eta=$ $\exp \left[\sum_{\mathbf{r}} \Theta(\mathbf{r}) \rho(\mathbf{r})\right] \exp \left[-\sum_{\mathbf{r}} \Theta(\mathbf{r}) \bar{\rho}\right]$, we focus on the first factor (will be denoted by $\eta$ for simplicity). To this end, we first consider

$$
\begin{aligned}
& \eta=: \xi^{\alpha}, \quad \xi:=\exp \left[\sum_{\mathbf{r}} \ln \phi(\mathbf{r}) \rho(\mathbf{r})\right], \\
& \theta(\mathbf{r})=:[\phi(\mathbf{r})]^{\alpha} .
\end{aligned}
$$

Note that in real space,

$$
\xi \psi_{i}^{\dagger}(\mathbf{r})=\phi(\mathbf{r}) \psi_{i}^{\dagger}(\mathbf{r}) \xi
$$

In the momentum basis,

$$
\xi \psi_{i}^{\dagger}(\mathbf{k})=\sqrt{V}^{-1} \sum_{\mathbf{r}} \psi_{i}^{\dagger}(\mathbf{r}) \phi(\mathbf{r}) e^{+i \mathbf{r} \cdot \mathbf{k}} \xi
$$

For our choice of $\theta(\mathbf{r})$ and $\phi(\mathbf{r})$,

$$
\begin{aligned}
\xi \psi_{i}^{\dagger}(\mathbf{k})= & {\left[+2 d \psi_{i}^{\dagger}(\mathbf{k})\right.} \\
& \left.-\sum_{\mu}\left(\psi_{i}^{\dagger}\left(\mathbf{k}+\hat{\mathbf{k}}_{\mu}\right)+\psi_{i}^{\dagger}\left(\mathbf{k}-\hat{\mathbf{k}}_{\mu}\right)\right)\right] \xi \\
= & : \sum_{\mathbf{k}} \psi_{i}^{\dagger}\left(\mathbf{k}^{\prime}\right) T\left(\mathbf{k}^{\prime}, \mathbf{k}\right)
\end{aligned}
$$

where $\hat{\mathbf{k}}_{\mu}=(2 \pi) \hat{\mathbf{n}}_{\mu} / L$. The matrix $T\left(\mathbf{k}^{\prime}, \mathbf{k}\right)$ can be viewed as a discretized Laplacian (tight-binding Hamiltonian) on the dual lattice.

In the basis which diagonalizes $\mathcal{H}(\mathbf{k})$,

$$
\begin{aligned}
\xi \chi_{\hat{a}}^{\dagger}(\mathbf{k}) \xi^{-1} & =\sum_{\mathbf{k}} \chi_{\hat{b}}^{\dagger}\left(\mathbf{k}^{\prime}\right) T\left(\mathbf{k}^{\prime}, \mathbf{k}\right)\left\langle u_{\hat{b}}^{-}\left(\mathbf{k}^{\prime}\right) \mid u_{\hat{a}}^{-}(\mathbf{k})\right\rangle \\
& =: \sum_{\mathbf{k}} \chi_{\hat{b}}^{\dagger}\left(\mathbf{k}^{\prime}\right) \tilde{T}_{\hat{b} \hat{a}}\left(\mathbf{k}^{\prime}, \mathbf{k}\right) .
\end{aligned}
$$

The matrix $\tilde{T}_{\hat{a} \hat{b}}\left(\mathbf{k}, \mathbf{k}^{\prime}\right)$ (or $\left.\tilde{T}_{\hat{a} \hat{b}}^{\dagger}\left(\mathbf{k}, \mathbf{k}^{\prime}\right)\right)$ is the properly defined "Laplacian" in the "curved" momentum space. 
The expectation value of the non-local operator is then given by

$$
\begin{aligned}
& \langle\Phi|\xi| \Phi\rangle=\operatorname{Det}\left[\tilde{T}_{\hat{a} \hat{b}}\left(\mathbf{k}, \mathbf{k}^{\prime}\right)\right], \\
& \langle\Phi|\eta| \Phi\rangle=\operatorname{Det}\left[\tilde{T}_{\hat{a} \hat{b}}^{\alpha}\left(\mathbf{k}, \mathbf{k}^{\prime}\right)\right] .
\end{aligned}
$$

where Det is taken over the hatted indices as well as the momentum. We have thus related the geometric mean of the position operator squared to the spectral geometry defined for the Laplacian in the curved momentum space.

\section{1. path-integral representation}

For simplicity, we assume there is only one band occupied. In this case,

$$
\begin{aligned}
& \tilde{T}_{\hat{a} \hat{b}}\left(\mathbf{k}, \mathbf{k}^{\prime}\right):=T\left(\mathbf{k}, \mathbf{k}^{\prime}\right)\left\langle u_{\hat{a}}^{-}(\mathbf{k}) \mid u_{\hat{b}}^{-}\left(\mathbf{k}^{\prime}\right)\right\rangle \\
= & 2 d \delta_{\mathbf{k}^{\prime}, \mathbf{k}}-\sum_{\mu=1}^{d}\left[\delta_{\mathbf{k}^{\prime}, \mathbf{k}+\hat{\mathbf{k}}_{\mu}} U_{\mu}(\mathbf{k})+\delta_{\mathbf{k}^{\prime}, \mathbf{k}-\hat{\mathbf{k}}_{\mu}} U_{\mu}^{\dagger}(\mathbf{k})\right] \\
= & : 2 d I-X
\end{aligned}
$$

where we have assumed two bands $a= \pm 1$, and we fill the lower band, $\hat{a}=-$. The link variable $U_{\mu}(\mathbf{k})=\langle u(\mathbf{k}+$ $\left.\hat{\mathbf{k}}_{\mu}\right)|u(\mathbf{k})\rangle$ is decomposed into the amplitude (hopping) and phase (gauge field) parts as

$$
\begin{aligned}
t_{\mu}(\mathbf{k}) & =\left|\left\langle u\left(\mathbf{k}+\hat{\mathbf{k}}_{\mu}\right) \mid u(\mathbf{k})\right\rangle\right| \\
e^{A_{\mu}(\mathbf{k})} & =\frac{\left\langle u\left(\mathbf{k}+\hat{\mathbf{k}}_{\mu}\right) \mid u(\mathbf{k})\right\rangle}{\left|\left\langle u\left(\mathbf{k}+\hat{\mathbf{k}}_{\mu}\right) \mid u(\mathbf{k})\right\rangle\right|} .
\end{aligned}
$$

For an infinitesimal $\hat{\mathbf{k}}_{\mu}$, they are approximated as

$$
\begin{aligned}
t_{\mu}(\mathbf{k}) & \simeq \exp \left(-\frac{1}{2} g_{\mu \mu}(\mathbf{k}) \Delta k_{\mu} \Delta k_{\mu}\right) \\
e^{A_{\mu}(\mathbf{k})} & \simeq \exp \left(\mathcal{A}_{\mu}(\mathbf{k}) \Delta k_{\mu}\right)
\end{aligned}
$$

where $\mu$ is not summed,

In order to compute $\operatorname{Det} \tilde{T}=\operatorname{Det}(2 d I-X)$, we consider the following formal expansion in terms of $Y=$ $X /(2 d)$ :

$$
\begin{aligned}
\operatorname{Det}(I-Y) & =\exp [\operatorname{Tr} \ln (I-Y)] \\
& =\exp \left[-\sum_{n=1}^{\infty} \frac{1}{n} \operatorname{Tr}\left(Y^{n}\right)\right] .
\end{aligned}
$$

For a given $n$, the trace is represented as a sum over paths (loops):

$$
\begin{aligned}
\operatorname{Tr}\left(Y^{n}\right) & =\left(\frac{1}{2 d}\right)^{n} \sum_{p} \sum_{C_{p}} \prod_{C_{p}}^{\left|C_{p}\right|=n} U_{\mu}(k) \\
& =\left(\frac{1}{2 d}\right)^{n} \sum_{p} \sum_{C_{p}} \prod_{C_{p}}^{\left|C_{p}\right|=n} t_{\mu}(\mathbf{k}) e^{A_{\mu}(\mathbf{k})}
\end{aligned}
$$

where $p \in \mathrm{BZ}$ is an initial point for paths, $C_{p}$ represents a path starting from $p$ and going back to $p$ with $n$ steps, and $\prod_{C_{p}} U_{\mu}(k)$ is the path-ordered product. Thus, the expansion can be interpreted as a summation over closed loops. It should be noted that $\prod_{C_{p}} U_{\mu}(k)$ is actually does not depends on the initial point $p$ at which one starts to draw a path.

Thus,

$$
\begin{aligned}
& \ln \langle\Phi|\xi| \Phi\rangle=\ln \operatorname{Det} \tilde{T} \sim \sum_{n=1}^{\infty} \frac{1}{n}\left(\frac{1}{2 d}\right)^{n} \sum_{p} \sum_{C_{p}}^{\left|C_{p}\right|=n} e^{-S} . \\
& \text { where } \quad S=\frac{1}{2} \int_{C_{p}} g_{\mu \mu}(k) \Delta k_{\mu} \Delta k_{\mu}-\int_{C_{p}} \mathcal{A}_{\mu} \Delta k_{\mu} \cdot(50)
\end{aligned}
$$

We can see that for a smaller value of the metric $g_{\mu \nu}$, the non-local operator takes a smaller value. Especially the trivial metric $g_{\mu \nu}=0$ gives the minimum expectation value of the operator.

\section{BURES METRIC FOR TOPOLOGICAL INSULATORS AND SUPERCONDUCTORS}

In this section, we explore momentum space geometries and topologies of gapped phases, applying general formalism presented in the previous sections. In particular, we will consider examples of topological insulators/superconductors constructed in Ref. [10]. In Ref. [10], gapped Hamiltonians of Dirac type are systematically constructed, which realize topological insulators/superconductors in all symmetry classes and in all dimensions. For these, we will discuss the momentum space metric and the second cumulant of the position operators, by following the Marzari-Vanderbilt formalism, and also by following the formalism developed in Sec. III B.

We will also discuss topological invariants made of the Bures metric, such as the Euler class $(d=2 n)$, the Pontrjagin class $(d=4 n)$, the gravitational Chern-Simons invariant $(d=2 n+1)$, and the dimensionally continued Euler density $(d=2 n+1)$. The dimensionally continued Euler densities have the following forms

$$
\Omega^{\mu_{1} \cdots \mu_{2 n}} \wedge E^{\mu_{2 n+1} \cdots \mu_{d}} \epsilon_{\mu_{1} \cdots \mu_{2 n}}
$$

where $\Omega^{\mu_{1} \cdots \mu_{2 n}} \equiv \Omega^{\mu_{1} \mu_{2}} \wedge \cdots \Omega^{\mu_{2 n-1} \mu_{2 n}}$ is the wedge product of the curvature two form and $E^{\mu_{2 n+1} \cdots \mu_{d}} \equiv$ $E^{\mu_{2 n+1}} \wedge \cdots E^{\mu_{d}}$ is the wedge product of the co-frames of the orthonormal frames. Among the dimensionally continued Euler densities, there is one which is a topological invariant for a given dimension $d$.

Following Ref. [10], we start with topological Dirac insulators (Sec. IV A) in $d=2 n$ - and $d=(2 n+1)$ - dimensions and discuss the general properties of the momentum space metric. These Dirac representatives realize topological insulators/superconductors characterized by an integer topological invariant for all symmetry classes 
and for all dimensions (sometimes called "primary series"). We will then focus on specific examples of the Dirac representatives in $d=2,3,4$, which include the QHE, the QSHE, and the $3 \mathrm{D} \mathbb{Z}_{2}$ topological insulator.

\section{A. Dirac representatives: generalities}

1. odd space-time dimensions, $d=2 n$

We start with the $d=2 n$-dimensional topological Dirac insulator which is defined in momentum space by

$$
\mathcal{H}_{(2 n+1)}^{d=2 n}(\mathbf{k})=\sum_{a=1}^{d=2 n} k_{a} \Gamma_{(2 n+1)}^{a}+m \Gamma_{(2 n+1)}^{2 n+1} .
$$

Here, $k_{a=1, \ldots, d}$ are a component of the $d$-dimensional momentum, and $\Gamma_{(2 n+1)}^{a=1, \ldots, 2 n+1}$ are $\left(2^{n} \times 2^{n}\right)$-dimensional hermitian matrices and satisfy $\left\{\Gamma_{(2 n+1)}^{a}, \Gamma_{(2 n+1)}^{b}\right\}=2 \delta_{a, b}$. The two eigenvalues of the Dirac Hamiltonian are

$$
\pm \lambda(k), \quad \lambda(k)=\sqrt{k^{2}+m^{2}},
$$

where $k=|\mathbf{k}|$.

The Dirac Hamiltonian (52) represents the topological insulator/superconductor in class A $(d=2 n)$, which is characterized by lack of any discrete symmetry, and by an integer topological invariant. For a given $d=2 n$, the Hamiltonian (52) respects either one of TRS or PHS. For this reason, the Dirac Hamiltonian (52) also represents the topological insulator/superconductor in class AI $(d=$ $8 m)$, class D $(d=8 m+2)$, class AII $(d=8 m+4)$, class $\mathrm{C}(d=8 m+6)$, where $m \in \mathbb{Z}$.

The momentum space metric for the Dirac Hamiltonian (52) is given, in the Cartesian and in the radial coordinates, by

$$
\begin{aligned}
d s^{2} & =\frac{2^{n}}{8 \lambda^{4}}\left(\delta_{a b} \lambda^{2}-k_{a} k_{b}\right) d k_{a} d k_{b} \\
& =\frac{2^{n}}{8}\left[\frac{m^{2}}{\lambda^{4}} d k^{2}+\frac{k^{2}}{\lambda^{2}} d \Omega_{d-1}\right]
\end{aligned}
$$

respectively. Here, $d s^{2}$ is the line element in momentum space, and $d \Omega_{d-1}$ is the line element of the $(d-1)$ dimensional unit sphere.

Near the origin $k=0$, and near $k=\infty$, the metric is given by

$$
d s^{2} \simeq \begin{cases}\frac{2^{n}}{8 m^{2}}\left(d k^{2}+k^{2} d \Omega_{d-1}\right), & k \rightarrow 0 \\ \frac{2^{n}}{8}\left(m^{2} \frac{d k^{2}}{k^{4}}+d \Omega_{d-1}\right), & k \rightarrow \infty .\end{cases}
$$

The metric for $k \rightarrow 0$ is equivalent to that of a sphere, while the metric near $k=\infty$ can be brought into a form, with $z=1 / k, d s^{2} \simeq \frac{2^{n}}{8}\left(m^{2} d z^{2}+d \Omega_{d-1}\right)$. I.e., the metric of a flat geometry. Therefore the momentum space is smoothly capped at $k=0$ while it has a finite radius at $k=\infty(z=0)$. This shows that the geometry of momentum space, defined by the Bures metric, is a semisphere or a cigar.

a. regularization For the Dirac Hamiltonian (52), the behavior of the wavefunctions at high energies is nontrivial. This is signaled by the fact that the Chern-integer for $d=2 n$, when computed for the Dirac Hamiltonian (52), is not quantized in integer unit but in half-odd integer unit. In turn, this is closely related to the fact that the momentum space geometry detected by the Bures metric is that of a semisphere. For these reasons, while the Dirac Hamiltonian (52) is capable of describing a transition between two phases with different topological charge (as we tune $m$ ), it does not represent, in a well-defined fashion, a topological or a non-topological insulator by itself 10 .

If we wish, such non-trivial behavior of the wavefunction can be regularized, by modifying the Bloch Hamiltonian at large momentum, for example, by replacing the constant mass by

$$
m \rightarrow \widetilde{m}(k)=m-C k^{2}
$$

where $C$ is some constant. With the regularization, the $n$-th Chern integer is quantized in integer unit, $\mathrm{Ch}_{n}=$ 1,0 for $\operatorname{sgn} m= \pm \operatorname{sgn} C$, respectively $(d=2 n)$.

The metric for the regularized Dirac Hamiltonian is given by

$$
\begin{aligned}
d s^{2} & =\frac{2^{n}}{8}\left[\frac{1}{\lambda^{2}} d k_{a} d k_{a}+\frac{(4 m C-1)}{\lambda^{4}} k_{a} k_{b} d k_{a} d k_{b}\right] \\
& =\frac{2^{n}}{8}\left[\frac{\left(m+C k^{2}\right)^{2}}{\lambda^{4}} d k^{2}+\frac{k^{2}}{\lambda^{2}} d \Omega_{d-1}\right]
\end{aligned}
$$

where $\lambda(k)=\sqrt{k^{2}+\widetilde{m}^{2}(k)}$.

Near the origin $k=0$, the metric shows the same asymptotic behavior, $d s^{2} \simeq \frac{2^{n}}{8 m^{2}}\left(d k^{2}+k^{2} d \Omega_{d-1}\right)$, as in the unregularized case, while near $k=\infty$,

$$
\begin{aligned}
d s^{2} & \simeq \frac{2^{n}}{8 C^{2}}\left(\frac{d k^{2}}{k^{4}}+\frac{1}{k^{2}} d \Omega_{d-1}\right) \\
& =\frac{2^{n}}{8 C^{2}}\left(d z^{2}+z^{2} d \Omega_{d-1}\right) .
\end{aligned}
$$

Therefore momentum space is smoothly capped both at $k=0$ and at $k=\infty(z=0)$. This shows that the topology of momentum space is a sphere.

It is also interesting to see the difference between the regularized and unregularized models at criticality. In the limit $m \rightarrow 0$, the metric for the unregularized model (54) reduces to $d s^{2}=\frac{2^{n}}{8} d \Omega_{d-1}$, i.e, $g_{k k}=0$. On the other hand, for the regularized model, (57), the metric reduces

$$
d s^{2} \rightarrow \frac{2^{n}}{8}\left[\frac{C^{2} d k^{2}}{\left(1+C^{2} k^{2}\right)^{2}}+\frac{d \Omega_{d-1}}{1+C^{2} k^{2}}\right] .
$$

Notice that the coefficient of $d \Omega_{d-1}$ does not vanish at $k=0$. Therefore, the topology of momentum space changes to create a new "hole" at the origin. 
Alternatively, one can put the theory on a lattice to regularize. This amounts to consider the following lattice version of the Dirac Hamiltonian:

$$
\mathcal{H}_{(2 n+1)}^{d=2 n}(\mathbf{k})=\sum_{a=1}^{d=2 n} \sin k_{a} \Gamma_{(2 n+1)}^{a}+f(\mathbf{k}) \Gamma_{(2 n+1)}^{2 n+1} .
$$

where $k_{a} \in(-\pi,+\pi]$, and $f(\mathbf{k})=(m+d)-$ $2 C \sum_{a=1}^{d=2 n} \cos k_{a}$. The eigenvalues are given by $\pm \lambda(\mathbf{k})$ with

$$
\lambda(\mathbf{k})=\sqrt{\sum_{a}\left(\sin k_{a}\right)^{2}+f^{2}(\mathbf{k})} .
$$

By expanding (60) near $\mathbf{k} \sim 0$, one recovers the Dirac Hamiltonian in the continuum, (52) and (56). The lattice Dirac Hamiltonian (60) will be used to discuss the expectation value of the non-local operator $\eta$. The metric in the Cartesian coordinates is given by

$$
\begin{aligned}
g_{a b}(\mathbf{k})= & \frac{2^{n}}{8 \lambda^{2}}\left[\cos k_{a} \cos k_{b} \delta_{a b}+4 C^{2} \sin k_{a} \sin k_{b}\right] \\
& -\frac{2^{n}}{8 \lambda^{4}} \sin k_{a} \sin k_{b} \\
& \times\left[\cos k_{a}+2 C f(\mathbf{k})\right]\left[\cos k_{b}+2 C f(\mathbf{k})\right],
\end{aligned}
$$

where repeated indices are not summed over. Observe that the volume element $\sqrt{g}$ can be zero, say, at $k_{a}=$ $\pm \pi / 2$.

\section{2. even space-time dimensions, $d=2 n-1$}

A Dirac Hamiltonian in even space-time dimensions $(d=2 n-1)$ can be obtained from (52) by formally replacing one component of the momentum by a mass term, $k_{2 n} \rightarrow m_{1}$ (dimensional reduction),

$$
\begin{aligned}
\mathcal{H}_{(2 n+1)}^{d=2 n-1}(\mathbf{k})= & \sum_{a=1}^{d=2 n-1} k_{a} \Gamma_{(2 n+1)}^{a} \\
& +m_{1} \Gamma_{(2 n+1)}^{2 n}+m_{2} \Gamma_{(2 n+1)}^{2 n+1} .
\end{aligned}
$$

When one of the masses is set to zero, the Hamiltonian anticommutes with $\Gamma_{(2 n+1)}^{2 n, 2 n+1}$, and this is a topological insulator in class AIII, i.e., there is chiral (sublattice) symmetry In addition to this chiral symmetry, for a given $d=2 n+1$, the Hamiltonian (63) respects either one of TRS or PHS. For this reason, the Dirac Hamiltonian 63) also represents the topological insulator/superconductor in class BDI $(d=8 m+1)$, class DIII $(d=8 m+3)$, class CII $(d=8 m+5)$, class CI $(d=8 m+7)$, where $m \in \mathbb{Z}$.

With the Dirac representatives (52) and (63), we cover all topological insulators/superconductors labeled by an integer topological invariant (called "primary series"). Topological insulators/superconductors characterized by an binary topological invariant $\left(\mathbb{Z}_{2}\right)$ or an even integral topological invariant $(2 \mathbb{Z})$ can be derived from these by dimensional reduction. We will not give an exhaustive study of the momentum space metric for these "descendents" (i.e., topological insulators/superconductors characterized either by $\mathbb{Z}_{2}$ or $2 \mathbb{Z}$ topological invariant). Rather, we will focus on the QSHE (class AII in $d=2$ ) and the $3 \mathrm{D} \mathbb{Z}_{2}$ topological insulator (class AII in $d=3$ ) (see below).

The two eigenvalues of the Dirac Hamiltonian (63) is

$$
\pm \lambda(k), \quad \lambda(k)=\sqrt{k^{2}+m_{1}^{2}+m_{2}^{2}}
$$

The calculations of the metric, etc., go parallel with the case of $d=2 n$. The quantum metric is given by

$$
g_{a b}(\mathbf{k})=\frac{2^{n}}{8 \lambda^{4}}\left(\delta_{a b} \lambda^{2}-k_{a} k_{b}\right)
$$

This is the same metric as that of the $d=2 n$ case. Therefore the geometry of momentum space is a cigar.

As before, non-trivial behaviors of the Bloch wavefunction can be regularized, by replacing the constant mass by

$$
m_{1,2} \rightarrow \widetilde{m}_{1,2}(k)=m_{1,2}-C_{1,2} k^{2} .
$$

With the regularization, the metric can be written

$$
\begin{aligned}
d s^{2}= & \frac{2^{n}}{8 \lambda^{2}} d k_{a} d k_{a}+\frac{2^{n}}{8 \lambda^{2}} k_{a} k_{b} \\
& \times\left[4|\vec{C}|^{2}|\vec{m}|^{2}-(2 \vec{m} \cdot \vec{C}-1)^{2}\right] d k_{a} d k_{b} \\
= & \frac{2^{n}}{8 \lambda^{4}}\left[\lambda^{2}+4|\vec{C}|^{2}|\vec{m}|^{2} k^{2}-k^{2}(2 \vec{m} \cdot \vec{C}-1)^{2}\right] d k^{2} \\
& +\frac{2^{n}}{8 \lambda^{2}} k^{2} d \Omega_{d-1}
\end{aligned}
$$

where $\lambda(k)=\sqrt{k^{2}+\widetilde{m} \cdot \widetilde{m}}$, and we have introduced a vectorial notation, for instance, $\vec{C} \cdot \vec{m}=C_{1} m_{1}+C_{2} m_{2}$, $|\vec{C}|^{2}=\vec{C} \cdot \vec{C}$, etc.

The structures of the metric at $k=0$ and $k=\infty$ are the same as those of $d=2 n$ case. Therefore, the topology of the momentum space is a sphere.

\section{B. example in $d=2$ : the IQHE (class A)}

Consider three mutually anticommuting, hermitian matrices $\Gamma_{(3)}^{a=1,2,3}=\left\{\sigma_{x}, \sigma_{y}, \sigma_{z}\right\}$, where $\sigma_{x, y, z}$ are the $2 \times 2$ Pauli matrices. We consider the following (unregularized) $d=2$-dimensional massive Dirac Hamiltonian,

$$
\mathcal{H}_{(3)}^{d=2}(\mathbf{k})=k_{x} \sigma_{x}+k_{y} \sigma_{y}+m \sigma_{z}
$$

This is a class A Hamiltonian.

Assuming $m>0$, the Berry connection and the Berry curvature are given by

$$
\begin{aligned}
& A_{x}(\mathbf{k})=+\frac{i k_{y}}{2 \lambda(\lambda+m)}, \quad A_{y}(\mathbf{k})=-\frac{i k_{x}}{2 \lambda(\lambda+m)}, \\
& F_{x y}(\mathbf{k})=\partial_{k_{x}} A_{y}-\partial_{k_{y}} A_{x}=-\frac{i m}{2 \lambda^{3}} .
\end{aligned}
$$


The Chern number is non-zero,

$$
\mathrm{Ch}_{1}=\frac{i}{2 \pi} \int d^{2} k F_{x y}=\frac{1}{2} \frac{m}{|m|},
$$

which is nothing but the Hall conductivity $\sigma_{x y}$. This model can be realized in the low energy limit of the honeycomb lattice model introduced by Haldane [28]. While the Chern integer in the unregularized model (68) in quantized in half-integer unit, with regularization, it is quantized in integer unit.

a. the Marzari-Vanderbilt cumulant We now discuss quantities constructed from the momentum space metric. Let us first discuss the Marzari-Vanderbilt cumulant $\Omega_{I, \mu \nu}$. Plugging the concrete form of the metric, we obtain, for the diagonal component,

$$
\begin{aligned}
& \Omega_{I, x x}=\Omega_{I, y y} \\
& =\frac{1}{16 \pi}\left[\frac{(2 C m-1) k^{2}-2 m^{2}}{2\left(C^{2} k^{4}-(2 C m-1) k^{2}+m^{2}\right)}\right. \\
& \left.+\frac{2 C m+1}{\sqrt{4 C m-1}} \arctan \left(\frac{2 C^{2} k^{2}-2 C m+1}{\sqrt{4 C m-1}}\right)\right]\left.\right|_{k=0} ^{k=\infty} \\
& \rightarrow \frac{1}{16 \pi} \\
& +\frac{1}{32} \frac{2 C m+1}{\sqrt{4 C m-1}}\left[1-\arctan \left(\frac{-2 C m+1}{\sqrt{4 C m-1}}\right)\right]
\end{aligned}
$$

while the off-diagonal component is vanishing $\Omega_{I, x y}=0$.

The behavior $\Omega_{I, x x}$ as a function of the mass parameter $m$ is shown in Fig. 11. Observe that the plot is not symmetric about $m=0$ : the cumulant for the positive mass $m>0$ is larger than for the negative mass $m<0$. This shows that the electrons tend to be delocalized in the topological phase ( $m>0$ in this case). In particular, the cumulant goes to zero as $m \rightarrow-\infty$, while it increases for positive $m$. This behavior should be compared with the lattice calculation in Refs. 21, 29], where the cumulant remains finite in the topological phase. Had we not regularized the Dirac model (68), $\Omega_{I, x x}$ as a function of the mass parameter $m$ would be symmetric with respect to $m=0$. At the critical point $m=0$, the cumulant diverges logarithmically

$$
\Omega_{I, x x} \sim \log m .
$$

b. topological invariant The topological number for a closed 2 dimensional manifold is the Euler number

$$
\chi=\frac{1}{4 \pi} \int d^{2} k \sqrt{g} R,
$$

which is equal to $2-2 g-b$ for a smooth manifold with genus $g$ and boundary number $b$.

As we mentioned above, the momentum space is a cigar and non-compact if we do not introduce a regulator. With the regularization $m \rightarrow \widetilde{m}(k)=m-C k^{2}$, the topology of the momentum space is a sphere and so the Euler number is $\chi=2$. Note that when $\operatorname{sgn}(m)=-\operatorname{sgn}(C)$, the metric (57) has a coordinate singularity at $k^{2}=-m / C$.

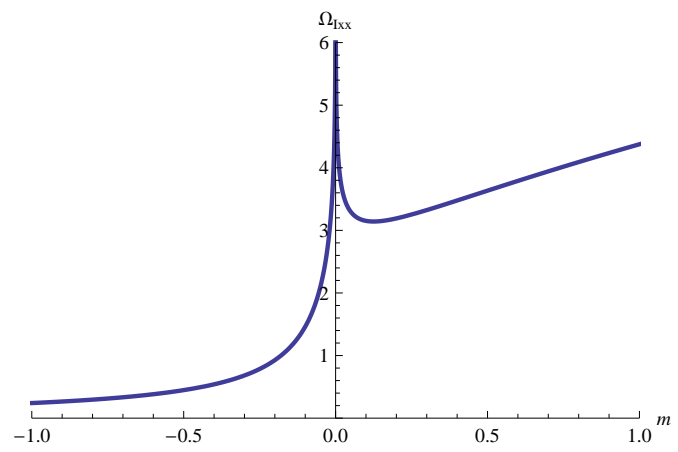

FIG. 1: The Marzari-Vanderbilt cumulant $\Omega_{I, x x}$ for the $2 \mathrm{D}$ Dirac model (68) as a function of the mass parameter $m$. The mass term is regularized by replacing $m$ in (68) by $m \rightarrow$ $\tilde{m}(k)=m-C k^{2}$, and we have set $C=1$.

c. second cumulant: the geometric mean We now study the expectation value of the non-local operator $\eta$ for a topological Dirac insulator in class A. Here, we considered the lattice regularized model (60) with $d=2$. The Dirac model describes the chiral $p$-wave superconductor ( $p$-wave $\mathrm{SC}$ ) on the square lattice defined by the tight-binding Hamiltonian

$$
\begin{aligned}
H= & \sum_{\mathbf{r}} \psi(\mathbf{r})^{\dagger}\left(\begin{array}{cc}
t & \Delta \\
-\Delta & -t
\end{array}\right) \psi(\mathbf{r}+\hat{\mathbf{x}})+\text { h.c. } \\
& +\psi(\mathbf{r})^{\dagger}\left(\begin{array}{cc}
t & i \Delta \\
i \Delta & -t
\end{array}\right) \psi(\mathbf{r}+\hat{\mathbf{y}})+\text { h.c. } \\
& +\psi(\mathbf{r})^{\dagger}\left(\begin{array}{cc}
\mu & 0 \\
0 & -\mu
\end{array}\right) \psi(\mathbf{r}),
\end{aligned}
$$

where the multi-component fermion annihilation operator at site $\mathbf{r}, \psi(\mathbf{r})$, is given in terms of the electron annihilation operators with spin up and down, $c_{\uparrow, \downarrow}(\mathbf{r})$, as $\psi^{T}(\mathbf{r})=\left(c_{\uparrow}(\mathbf{r}), c_{\downarrow}^{\dagger}(\mathbf{r})\right)$, and we take $t=\Delta=1$ and $\mu=m+2$. The chiral $p$-wave SC has been discussed in the context of superconductivity in a ruthenate and paired states in the fractional quantum Hall effect 30 35. There are four phases separated by three quantum critical points at $\mu=0, \pm 4$, which are labeled by the Chern number as $\mathrm{Ch}=0(|\mu|>4), \mathrm{Ch}=-1$ $(-4<\mu<0)$, and $\mathrm{Ch}=+1(0<\mu<+4)$. The non-zero Chern number implies the IQHE in the spin transport 32].

In Fig. 2, we plot the expectation value of the nonlocal operator, $\alpha^{-1} \ln \langle\Phi|\eta| \Phi\rangle$, obtained by numerically diagonalizing the Laplacian operator in momentum space $\tilde{T}_{\hat{a} \hat{b}}\left(\mathbf{k}, \mathbf{k}^{\prime}\right), \quad V^{-1} \ln \langle\Phi|\eta| \Phi\rangle=V^{-1} \ln \operatorname{Det}\left[\tilde{T}_{\hat{a} \hat{b}}\left(\mathbf{k}, \mathbf{k}^{\prime}\right)\right]$. The behavior of $V^{-1} \ln \langle\Phi|\eta| \Phi\rangle$ as a function of $\mu$ is qualitatively similar to the Marzari-Vanderbilt cumulant: (i) it is larger in the topological phase $(-4<\mu<4)$ than in the non-topological phases $(|\mu|>4$ ), and (ii) near the critical points $\mu= \pm 4,0$, it shows a peak structure. Due to the system size limit accessible by numerics, it is admittedly difficult to see if $V^{-1} \ln \langle\Phi|\eta| \Phi\rangle$ develops a 


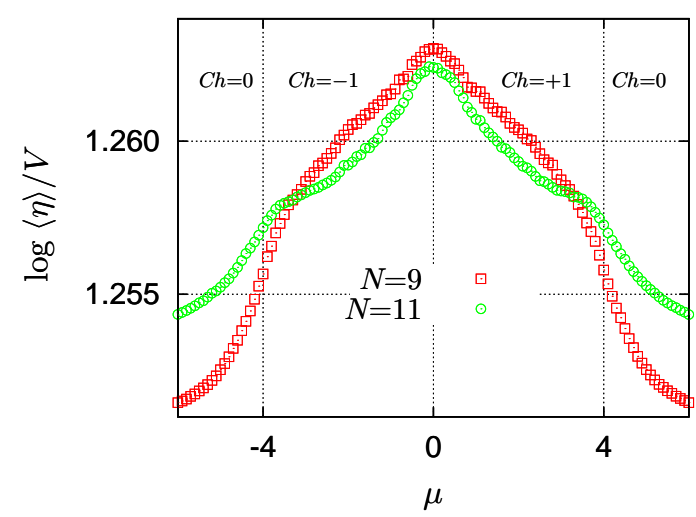

FIG. 2: The expectation value of the non-local operator $V^{-1} \ln \langle\Phi|\eta| \Phi\rangle$ for the lattice Dirac Hamiltonian (lattice chiral $p$-wave superconductor) (74) as a function of $\mu$.

singularity at the critical points.

Some technical comments are in order. Computing $V^{-1} \ln \langle\Phi|\eta| \Phi\rangle$ by numerically diagonalization the momentum space Laplacian, and then summing over logarithm of eigenvalues can be quite tricky, since some eigenvalues are very close to zero. In order to obtain a meaningful result, we can modify (or regularize) $\phi(\mathbf{r})$ as

$$
\begin{aligned}
\phi(\mathbf{r}) & =-2 \sum_{\mu=1}^{d} \cos \frac{2 \pi n_{\mu}}{N}+2 d \\
& \rightarrow-2 \sum_{\mu=1}^{d} \cos \frac{2 \pi\left(n_{\mu}+\delta n_{\mu}\right)}{N}+2(d+\delta d) .
\end{aligned}
$$

Here, adding $\delta d \ll d$ shifts all eigenvalues $\varepsilon_{i}$ of the Laplacian, $\varepsilon_{i} \rightarrow \varepsilon+2 \delta d$, thereby prevents having an eigenvalue which is too close to zero. The expectation value $\ln \operatorname{Det}\left[\tilde{T}_{\hat{a} \hat{b}}\left(\mathbf{k}, \mathbf{k}^{\prime}\right)\right]$ can be evaluated for small but finite $\delta d$, and the results can be interpolated to $\delta d \rightarrow 0$. Adding $0<\delta n_{\mu}<1$ amounts shifting the "location" of the nonlocal operator at which it is inserted; the function $\phi(\mathbf{r})$ is centered at a site $\mathbf{r}=0$, but alternatively, one can define $\phi(\mathbf{r})$ in such a way that it is centered at the center of a plaquette (i.e., a dual site), $\delta n_{\mu}=1 / 2$. Or, more generally, one can take the center of $\phi(\mathbf{r})$ anywhere in a plaquette, $0<\delta n_{\mu}<1$. In Fig. 2, the expectation value $\ln \operatorname{Det}\left[\tilde{T}_{\hat{a} \hat{b}}\left(\mathbf{k}, \mathbf{k}^{\prime}\right)\right]$ is averaged over different centers (different choice of $0<\delta n_{\mu}<1$ ) to obtain smooth curves.

\section{C. example in $d=2$ : the QSHE (class AII)}

Consider five mutually anticommuting, hermitian matrices

$$
\Gamma_{(5)}^{a=1, \ldots, 5}=\left\{\alpha_{x}, \alpha_{y}, \alpha_{z}, \beta,-i \beta \gamma^{5}\right\}
$$

where we are using the Dirac representation,

$$
\alpha_{i}=\left(\begin{array}{cc}
0 & \sigma_{i} \\
\sigma_{i} & 0
\end{array}\right), \beta=\left(\begin{array}{cc}
1 & 0 \\
0 & -1
\end{array}\right), \gamma^{5}=\left(\begin{array}{ll}
0 & 1 \\
1 & 0
\end{array}\right) .
$$

Observe that $\alpha_{x} \alpha_{y} \alpha_{z} \beta=-i \beta \gamma_{5}$. We will use these gamma matrices to construct Dirac representative for $d=2,3,4$ below.

Let us consider the following $d=2$-dimensional Dirac Hamiltonian.

$$
\begin{aligned}
\mathcal{H}_{(5)}^{d=2}(\mathbf{k}) & =k_{x} \alpha_{1}+k_{y} \alpha_{2}+\alpha \gamma_{5}+\widetilde{m}(k)\left(-i \beta \gamma_{5}\right), \\
\widetilde{m}(k) & =m-C k^{2} .
\end{aligned}
$$

This Hamiltonian is time reversal invariant,

$$
T \mathcal{H}^{*}(-\mathbf{k}) T^{-1}=\mathcal{H}(\mathbf{k}), \quad T=i \tau_{x} \otimes \sigma_{y} .
$$

The Dirac Hamiltonian of type (78) was used in Refs.

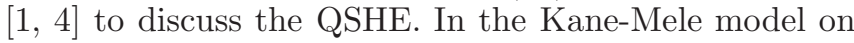
the honeycomb lattice, the mass $m$ is generated by a time-reversal spin-dependent second neighbor hopping, whereas the $\alpha$-term arises from the Rashba spin-orbit coupling. Time reversal invariance is crucial in the QSHE. When the edge states consist of even number of Kramers' pairs of counter propagating modes (helical modes), they can be gapped under perturbations. On the other hand, when the number of helical modes is odd, they cannot be gapped unless time reversal symmetry is broken.

The eigenvalues of the hamiltonian (78) are

$$
\begin{aligned}
E(k) & = \pm \lambda_{k}^{+}, \pm \lambda_{k}^{-} \\
\lambda_{k}^{ \pm} & =\sqrt{(k \pm \alpha)^{2}+\left(m-C k^{2}\right)^{2}}
\end{aligned}
$$

Notice that for $\alpha=0$, there is a critical point at $m=0$ and $k=0$. For $\alpha>0, m / C>0$, there is a critical point at $m / C=\alpha^{2}$ and $k=\sqrt{m / C}=\alpha$. As observed in Refs. [36, 37, there is a metallic phase near $m=0$ for $\alpha \neq 0$ sandwiched by insulating phases, while for $\alpha=0$, the metallic phase shrinks to a critical point.

The Bures metric is given by

$$
\begin{aligned}
& d s^{2} \\
= & \frac{1}{4}\left[\frac{(m+C k(k-2 \alpha))^{2}}{A_{-}(k)^{2}}+\frac{(m+C k(k+2 \alpha))^{2}}{A_{+}(k)^{2}}\right] d k^{2} \\
& +\frac{1}{4}\left[1+\frac{k^{2}-\alpha^{2}-\left(m-C k^{2}\right)^{2}}{\sqrt{A_{+}(k) A_{-}(k)}}\right] d \varphi^{2} .
\end{aligned}
$$

where

$$
A_{ \pm}(k):=\left(m-C k^{2}\right)^{2}+(k \pm \alpha)^{2} .
$$

Observe that $g_{k k}$ is a non-negative function as seen from (81), while the behavior of $g_{\varphi \varphi}$ is different for topologically trivial and topologically non-trivial phases; $g_{\varphi \varphi}$ is zero at $k=\sqrt{m / C}$ if $-\frac{m}{C}+\alpha^{2}>0$. 


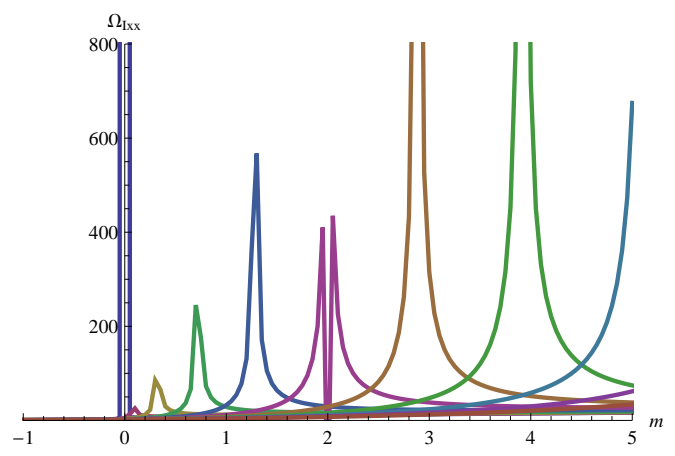

FIG. 3: The Marzari-Vanderbilt cumulant $\Omega_{I, x x}\left(=\Omega_{L_{-y y}}\right)$ for the two-dimensional $\mathbb{Z}_{2}$ topological Dirac insulator $(78)$ as a function of the mass parameter $m$. Here, $C=2$, and $\alpha=$ $0,0.2,0.4,0.6,0.8,1,1.2,1.4,1.6$. from the left to the right.

Let us first discuss several asymptotic behaviors of the metric. We first consider the case of $m \neq 0$. Near the origin $k=0$, the metric is given by

$$
d s^{2}=\frac{m^{2}}{2\left(m^{2}+\alpha^{2}\right)^{2}}\left(d k^{2}+k^{2} d \varphi^{2}\right)+\cdots .
$$

On the other hand, asymptotic value of $g_{k k}(k \rightarrow \infty)$ is $\frac{1}{2 C^{2} k^{4}}$. By making a coordinate transformation $z=$ $1 /(\sqrt{2} C k)$, the metric is given by

$$
d s^{2}=d z^{2}+z^{2} d \varphi^{2}+\cdots .
$$

Both (83) and (84) are the metric of a sphere, and therefore we see that the momentum space manifold is smoothly capped both at the origin and the infinity.

On the other hand, when $m=0$ and $\alpha \neq 0$, the metric near the origin $k=0$ is

$$
d s^{2}=\frac{C^{2}}{2 \alpha^{2}}\left(d\left(k^{2}\right)^{2}+\frac{\left(k^{2}\right)^{3}}{\alpha^{2}} d \varphi^{2}\right)+\cdots .
$$

This metric has a singularity at the origin. This should be compared with the case of $m=0$ and $\alpha=0$, for which the metric near the origin is

$$
d s^{2}=\frac{C^{2}}{2} d k^{2}+\frac{1}{2} d \varphi^{2} .
$$

The $\varphi$ circle does not shrink to zero at the origin and the metric is analytically continued to the positive energy region. Note that the energy becomes zero at $k=0$ and the gap is closed.

d. the Marzari-Vanderbilt cumulant The MarzariVanderbilt second cumulant is calculated numerically in Fig. 3. At the critical point $\alpha=m=0$, the cumulant diverges. For finite $\alpha$, the cumulant is finite with a peak at at $m=C \alpha^{2}$, whose peak value increases as $\alpha$ increases. In another critical case (pink line in Fig. 3) where the radial direction of the momentum space shrinks to zero at certain value of $k$, the cumulant gets two peaks and at the critical point $m=C \alpha^{2}(=2$ in Fig. 3), the cumulant takes small but finite value. The cumulant changes very rapidly around the critical point $m=C \alpha^{2}$. Above this value $\alpha=1$, the cumulant monotonically increases. Another important property of the cumulant is that for fixed $\alpha$, the cumulant takes larger value in $m-C \alpha^{2}>0$. As studied in [9, 37], $m-C \alpha^{2}>0$ region is the $\mathbb{Z}_{2}$ topological superconductor phase. Therefore, this shows that the electron tends to be delocalized in the topologically non-trivial phase.

D. examples in $d=3$ : chiral topological insulator (class AIII) and $\mathbb{Z}_{2}$ topological insulator (class AII)

As in the general discussion before, we can consider two mass terms in $d=3$,

$$
\mathcal{H}_{(5)}^{d=3}(\mathbf{k})=\sum_{a=1}^{3} k_{a} \Gamma_{(5)}^{a}+m_{1} \Gamma_{(5)}^{4}+m_{2} \Gamma_{(5)}^{5} .
$$

When either one of the masses are switched off, this Hamiltonian belongs to the symmetry class AIII, or AII.

As discussed in Ref. [7] for class AII and in Ref. [38] for class AIII, non-trivial topology of the Dirac Hamiltonian is signaled by the existence of the $\theta$-term in the effective action for the linear electromagnetic response with $\theta=\pi$. The non-zero (and quantized) $\theta$-term, in turn, is related to the topological invariant made of the Berry connection, which is given for the Dirac model (87),

$$
\begin{aligned}
\mathrm{CS}_{3} & =\frac{-1}{8 \pi^{2}} \int d^{3} k \epsilon^{\mu \nu \rho} \operatorname{tr}\left(A_{\mu} \partial_{\nu} A_{\rho}+\frac{2}{3} A_{\mu} A_{\nu} A_{\rho}\right) \\
& =\frac{1}{\pi} \frac{m_{2}}{\left|m_{2}\right|} \arctan \left[\frac{\left(m_{2}^{2}+m_{1}^{2}\right)^{1 / 2}-m_{1}}{\left(m_{2}^{2}+m_{1}^{2}\right)^{1 / 2}+m_{1}}\right]^{1 / 2} .
\end{aligned}
$$

In particular, when $m_{1}=0, \mathrm{CS}_{3}=(1 / 4) m_{2} /\left|m_{2}\right|$. As the CS term is determined only modulo 1 , the fractional part $(1 / 4) \operatorname{sgn}\left(m_{2}\right)$ is an intrinsic property. On a lattice, as there are two-copies of the $4 \times 4$ Dirac Hamiltonians, the total Chern-Simons invariant is given by $\mathrm{CS}_{3}=(1 / 2) \operatorname{sgn}\left(m_{2}\right)$, and $\exp \left(2 \pi i \mathrm{CS}_{3}\right)=-1$.

Let us now set $m_{1}=0$, and, as before, regularize the mass,

$$
\begin{aligned}
\mathcal{H}_{(5)}^{d=3}(\mathbf{k}) & =k_{x} \alpha_{1}+k_{y} \alpha_{2}+k_{z} \alpha_{3}+\widetilde{m}(k)\left(-i \beta \gamma_{5}\right), \\
\widetilde{m}(k) & =m-C k^{2} .
\end{aligned}
$$

This Hamiltonian can be obtained from the Dirac representative of the QSHE by replacing $\alpha$ in the two dimensional hamiltonian (78) by $k_{z}$.

The metric for the Dirac model (89) is

$$
\begin{aligned}
d s^{2}= & \frac{1}{2\left[\left(C k^{2}-m\right)^{2}+k^{2}\right]}\left[\frac{\left(C k^{2}+m\right)^{2}}{\left(C k^{2}-m\right)^{2}+k^{2}} d k^{2}\right. \\
& \left.+k^{2}\left(d \theta^{2}+\sin ^{2} \theta d \phi^{2}\right)\right]
\end{aligned}
$$




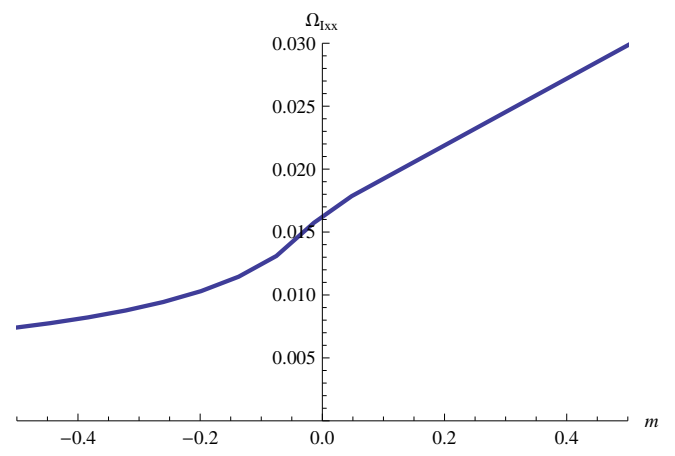

FIG. 4: The Marzari-Vanderbilt cumulant $\Omega_{I, x x}\left(=\Omega_{I, y y}\right)$ as a function of the mass parameter $m$ for for the 3D Dirac model (89) with $C=2$.

where we used the spherical coordinate, $\left(k_{x}, k_{y}, k_{z}\right)=$ $(k \sin \theta \cos \phi, k \sin \theta \sin \phi, k \cos \theta)$.

The behavior of the metric at $k=\infty$ is similar to that of the $d=2$ case. Here, we check the regularity of the metric at the origin. For $m \neq 0$, the metric is

$$
d s^{2} \cong \frac{1}{2 m^{2}}\left[d k^{2}+k^{2}\left(d \theta^{2}+\sin ^{2} \theta d \phi^{2}\right)\right]
$$

while for $m=0, C \neq 0$, it is

$$
d s^{2} \cong \frac{C^{2}}{2} d k^{2}+\frac{1}{2}\left(d \theta^{2}+\sin ^{2} \theta d \phi^{2}\right) .
$$

The momentum space is regular for finite $m$, while at the critical point $m=0$ there is a hole at the origin $k=0$. Note that when $\operatorname{sign}(C)=-\operatorname{sign}(m)$, there is a coordinate singularity $g_{k k}=0$ at $C k^{2}+m=0$. This suggests that the cumulant for $\operatorname{sign}(C)=-\operatorname{sign}(m)$ phase would take smaller value than that for $\operatorname{sign}(C)=\operatorname{sign}(m)$ phase. We will show this explicitly in the next paragraph.

e. the Marzari-Vanderbilt cumulant The diagonal component of the Marzari-Vanderbilt cumulant $\Omega_{I, x x}(=$ $\Omega_{I, y y}$ ) for the 3D Dirac Hamiltonian (89), obtained by numerically integrating the metric over three dimensional momentum space, is shown in Fig. 4 . The off-diagonal component is identically zero, $\Omega_{I, x y}=0$. The cumulant does not diverge at $m=0$, while its first derivative with respect to the mass $m$ changes discontinuously:

$$
\Omega_{I, x x}= \begin{cases}\frac{5}{48 \pi C}+\frac{5}{24 \pi} m+\cdots, & m \rightarrow-0 \\ \frac{5}{48 \pi C}+\frac{1}{12 \pi} m+\cdots, & m \rightarrow+0\end{cases}
$$

As mentioned above, the negative mass $(m<0)$ phase has a coordinate singularity $\left(g_{k k}=0\right)$ and the cumulant becomes smaller compared to the positive mass phase. The cumulant gets larger for large positive $m$. However, since this would come from the UV region of the integration, it would not have physical significance. f. topological invariants In $d=3$ momentum space, one can think of two invariants. Both of them are related to a Chern-Simons characteristics class, derived from the Pontrjagin, and the Euler characteristics classes, respectively.

The Chern-Simons invariant derived from the Pontrjagin class is the gravitational Chern-Simons term, which was discussed, for example, in Refs. [30, 39], and is given by

$$
I_{\mathrm{GCS}}=\frac{-1}{8 \pi^{2}} \int d^{3} k \operatorname{tr}\left(\omega d \omega+\frac{2}{3} \omega^{3}\right),
$$

where $\omega$ is a connection one form. This invariant turns out to be zero, since the integrand vanishes identically for the metric $(90)$.

Another invariant is the dimensionally continued Euler number. In three-dimensions, dimensionally continued Euler number can be written as

$$
\chi=\frac{1}{2(4 \pi)^{2}} \int \epsilon_{a b c} \Omega^{a b} \wedge e^{c}
$$

where $\epsilon_{a b c} \Omega^{a b} \wedge e^{c}=\epsilon_{a b c} R^{a b}{ }_{k l} e^{k} \wedge e^{l} \wedge e^{c}=2 R^{a b}{ }_{a b} \sqrt{g} d^{3} k$. For the unregularized model, the Dirac (89) with $C=0$, the dimensionally continued Euler number is given by

$$
\chi=\frac{3 \sqrt{2}}{4^{2}} .
$$

It is straightforward to check that this result still holds for the regularized model where $m_{2}$ is replaced by $\widetilde{m}_{2}(k)=m_{2}-C_{2} k^{2}$. When $m_{1}=0$ and $m_{2}=1$ with $C_{2}=1$, the integral can be evaluated analytically, and gives the same result as above.

\section{E. example in $d=4$}

The gamma matrices $(76)$ can be used to construct a $d=4$ dimensional Dirac Hamiltonian,

$$
\mathcal{H}_{(5)}^{d=4}(\mathbf{k})=\sum_{a=1}^{4} k_{a} \Gamma_{(5)}^{a}+m \Gamma_{(5)}^{5} .
$$

The Hamiltonian (97) is a topological insulator in class A with the non-zero second Chern number constructed with the Berry curvature [7, 40]. The Hamiltonian (97) respects time-reversal symmetry which squares to be -1, and hence belongs to symmetry class AII. The 3D Dirac Hamiltonian (87), which is a $\mathbb{Z}_{2}$ topological insulator in class AII, can be obtained from (97) by dimensional reduction. [7.

g. topological invariants The Euler number calculated by using the Bures metric is

$$
\chi=\frac{1}{128 \pi^{2}} \int d^{4} k \sqrt{g} \epsilon^{k l m n} \epsilon^{a b c d} R_{k l a b} R_{m n c d}=1 .
$$


On the other hand, the Pontrjagin number (the gravitational instanton term, or the signature $\tau$ ) is identically zero:

$$
P=\frac{1}{32 \pi^{2}} \int d^{4} k \sqrt{g} R_{m n l r} \epsilon^{m n a b} R_{a b}^{l r}=0 .
$$

\section{CONCLUDING REMARK AND DISCUSSION ON INTERACTION EFFECTS}

In this paper, we studied several properties of quantum ground states in momentum space by using the $\mathrm{Bu}-$ res metric. The Berry gauge potential and the Bures metric naturally appear in the expectation value of the non-local operator which measures the charge distributions. Namely, the Berry gauge potential measures polarization in real space and the Bures metric measures the second cumulant. These are the first two expansions of the generating function (24).

We introduced the distance square operator in real space which corresponds to the Laplacian in the curved momentum space. In the path integral description, this operator is given by the summation of geodesic lengths and phases of all closed loops in momentum space.

These operators, the second cumulant and the distance square operators, are applied to analyze some topological insulators. In certain phases, the Bures metrics possess the coordinate singularities where some components of the metric vanish. As a result, the cumulants take smaller values in those phases compared to other topologically non-trivial phases. These operators also capture the singular behaviors of the quantum phase transitions.

We also calculated several topological quantities such as the Euler number by using the Bures metric. These are analogous of the Chern number for the Berry gauge potential, which does characterize topological phases and has physical meaning. At this point, it is not very clear if the topological numbers for the Bures metric directly related to some observational quantities. The Euler number, for instance, measures the topologies of the momentum spaces. Naively these are spheres or tori depending on regularizations. However, we have seen in the case of the $2 d \mathbb{Z}_{2}$ topological insulator that the topology could be non-trivial: one cycle shrinks to zero at finite momentum $k$. Also, we have seen that there are several types of singularities in the momentum space geometries for both of the $\mathbb{Z}$ and $\mathbb{Z}_{2}$ insulators. We would leave the investigation of observational consequences of these phenomena for the future works.

Our analyses so far are based on free theories. It is interesting to see how the interaction effects change the story. In interacting systems, while the ground state cannot be decomposed as a product the Slater determinants as Eq. (8), the Berry connection and the Bures metric can be defined, in principle, by twisting the boundary conditions 41].

A yet another possible approach for interacting systems is to note that the spectral projector $Q(\mathbf{k})$ is nothing but the Fourier transform of the equal time correlation function $\left\langle\Phi\left|\psi_{i}^{\dagger}(\mathbf{r}) \psi_{j}(0)\right| \Phi\right\rangle$ 42]. A proper generalization of the momentum space metric in interacting systems can then be defined in terms of the equal time correlation function.

As an example, let us consider the $(2+1)$-dimensional Gross-Neveu model. I.e., the model with the Dirac kinetic term as in (68), and with a four-fermion interaction. To the leading order in the large- $N$ expansion (here, $N$ is the number of fermion flavors), the Euclidean fermion propagate is evaluated as (see, for example, Ref. 443])

$$
\mathcal{G}\left(k_{\mu}\right)=\frac{i \gamma_{\mu} k^{\mu}-m}{k^{2}+m^{2}}\left(\frac{\Lambda}{k}\right)^{a}
$$

where $\mu=0,1,2,3, k_{\mu=0,1,2,3}$ are the space-time momenta, $\Lambda$ is the ultra-violet cutoff, and $a=\mathcal{O}(1 / N)$.

The equal time correlator can be read off from $\mathcal{G}$ by $\int_{-\infty}^{+\infty} d k_{0} \mathcal{G}\left(k_{0}, k_{i}\right)=G\left(k_{i}\right)$. With the equal time correlator, we can compute the large- $N$ corrected quantum metric. The calculation amounts to modifying the projector $Q$ by $Q(\mathbf{k})=\mathcal{H}(\mathbf{k}) / \lambda(\mathbf{k}) \rightarrow Q(\mathbf{k})=\mathcal{H}(\mathbf{k}) / \tilde{\lambda}(\mathbf{k})$, where $\lambda(\mathbf{k})=\left(k^{2}+m^{2}\right)^{\frac{1}{2}}$, and $\tilde{\lambda}(\mathbf{k})=\left(k^{2}+m^{2}\right)^{\frac{1+\gamma}{2}}$. The exponent $\gamma$ is $\mathcal{O}(1 / N)$. The metric is then given in the radial coordinate by

$$
d s^{2}=\frac{2^{n}}{8}\left\{\left[\frac{1}{\lambda^{2+2 \gamma}}+\frac{\left(\gamma^{2}-1\right) k^{2}}{\lambda^{4+2 \gamma}}\right] d k^{2}+\frac{k^{2} d \Omega_{d-1}}{\lambda^{2+2 \gamma}}\right\} .
$$

In the limit $m \rightarrow 0$, this becomes

$$
d s^{2}=\frac{2^{n}}{8}\left[\frac{\gamma^{2}}{k^{2+2 \gamma}} d k^{2}+\frac{1}{k^{2 \gamma}} d \Omega_{d-1}\right] .
$$

The momentum space is singular at $k=0$ as in the case of non-interacting systems. However, the structure of the singularity is different. It would also be interesting to notice that in the absence of interaction, the metric is not deformed as we change the number of flavors $N$. On the other hand, in the interacting case, the metric is deformed as a function of $N$. This would also lead to deformation in the second cumulant.

Another way of considering the interacting systems is to look at string theory construction of the topological phases. As pointed out in 12 and constructed explicitly in [44], K-theory charges for the topological insulators (superconductors) are closely related to those of D-branes in string theory. Since D-brane systems are interacting ones by its construction, it would be interesting to consider momentum space geometries from D-brane point of view.

\section{Acknowledgments}

We acknowledge "Quantum Criticality and the AdS/CFT correspondence" miniprogram at KITP. We 
would like to thank J. Polchinski and H. Katsura for useful discussion. The research of SM was supported in part by the National Science Foundation under Grant
No. PHY05-51164 and JSPS. SR thanks the Center for Condensed Matter Theory at University of California, Berkeley for its support.
[1] C. L. Kane and E. J. Mele, Phys. Rev. Lett. 95, 146802 (2005); 95, 226801 (2005).

[2] R. Roy, Phys. Rev. B 79, 195321 (2009); ibid, 195322 (2009).

[3] J. E. Moore and L. Balents, Phys. Rev. B 75, 121306(R) (2007).

[4] B. A. Bernevig, T. L. Hughes, and S.-C. Zhang, Science 314, 1757 (2006).

[5] M. König et al., Science 318, 766 (2007).

[6] L. Fu, C. L. Kane, and E. J. Mele, Phys. Rev. Lett. 98, 106803 (2007).

[7] X.-L. Qi, T. Hughes, and S.-C. Zhang, Phys. Rev. B 78, 195424 (2008).

[8] D. Hsieh et al, Nature 452, 970 (2008).

[9] A. Schnyder, S. Ryu, A. Furusaki, A. Ludwig, Phys. Rev. B 78, 195125 (2008); AIP Conf. Proc. 1134, 10 (2009);

[10] S. Ryu, A. Schnyder, A. Furusaki, and A. Ludwig, arXiv:0912.2157.

[11] A. Kitaev, AIP Conf. Proc. 1134, 22 (2009).

[12] P. Horava, Phys. Rev. Lett. 95, 016405 (2005) [arXiv:hep-th/0503006].

[13] D. J. Thouless, M. Kohmoto, P. Nightingale, and M. den Nijs, Phys. Rev. Lett. 49, 405 (1982).

[14] M. Kohmoto, Ann. Phys. (N.Y.) 160355 (1985).

[15] L. Fu and C. L. Kane, Phys. Rev. B 74, 195312 (2006).

[16] N. Marzari, and D. Vanderbilt, Phys. Rev. B 56, 12847 (1997).

[17] I. Souza, T. Wilkens, and R. Martin, Phys. Rev. B 62, 1666 (2000).

[18] M. V. Berry, Proc. R. Soc. Lond. A 392, 45 (1984).

[19] F. Wilczek and A. Zee, Phys. Rev. Lett. 52, 2111 (1984).

[20] J. P. Provost and G. Vallee, Commun. Math. Phys. 76, 289 (1980).

[21] Yu-Quan Ma, Shu Chen, Heng Fan, and Wu-Ming Liu, arXiv:1003.4040 [quant-ph].

[22] R. Resta, Rev. Mod. Phys. 66, 899 (1994).

[23] R. Resta, Phys. Rev. Lett. 80, 1800 (1998).

[24] C. Sgiarovello, M. Peressi, and R. Resta, Phys. Rev. B
64, 115202 (2001).

[25] Ryuichi Shindou, Ken-Ichiro Imura, and Masao Ogata, Phys. Rev. B 74, 245107 (2006).

[26] D. H. Lee and S. A. Kivelson, Phys. Rev. B 67, 024506 (2003).

[27] S. Ryu, and Y. Hatsugai, Phys. Rev. B 73, 245115 (2006).

[28] F. D. M. Haldane, Phys. Rev. Lett 61, 2015 (1988).

[29] T. Thonhauser, and David Vanderbilt, Phys. Rev. B 74, 235111 (2006).

[30] N. Read and D. Green, Phys. Rev. B 61, 10267 (2000).

[31] G. E. Volovik, Pis'ma ZhETF, JETP Lett. 66, 493 (1997).

[32] T. Senthil, M. P. A. Fisher, L. Balents and C. Nayak, Phys. Rev. Lett. 81, 4704 (1998).

[33] J. Goryo and K. Ishikawa, Phys. Lett. A 260, 294 (1999).

[34] Y. Morita and Y. Hatsugai, Phys. Rev. B 62, 99 (2000).

[35] Y. Hatsugai and S. Ryu, Phys. Rev. B 65, 212510 (2002).

[36] H. Obuse, A. Furusaki, S. Ryu, and C. Mudry, Phys. Rev. B 76, 075301 (2007).

[37] S. Ryu, C. Mudry, H. Obuse, and A. Furusaki, arXiv:0912.2158.

[38] Pavan Hosur, Shinsei Ryu, and Ashvin Vishwanath, Phys. Rev. B, 81, 045120 (2010).

[39] S. Deser, R. Jackiw and S. Templeton, Annals Phys. 140, 372 (1982) [Erratum-ibid. 185, 406.1988 APNYA,185,406.1988 APNYA,281,409 (1988 APNYA,185,406.1988 APNYA,2]. Phys. Rev. Lett. 48, 975 (1982). S. Deser and R. Jackiw, Phys. Rev. Lett. 59, 1981 (1987).

[40] M. F. L. Golterman, K. Jansen, and D. B. Kaplan, Phys. Lett. B 301, 219 (1993)

[41] Qian Niu, D. J. Thouless, and Yong-Shi Wu, Phys. Rev. B 31, 3372 (1985).

[42] I. Peschel, J. Phys. A : Math. Gen. 36, L205 (2003).

[43] S. Hands, A. Kocic, and J. B. Kogut, Annals of Phys. 224, 29 (1993).

[44] S. Ryu and T. Takayanagi, arXiv:1001.0763 [hep-th]. 